\title{
Efficacy and Safety of Intravenous Nicorandil on blood pressure in patients with coronary heart disease Corresponging author: Yan He, Email :heyan20200418@126.com
}

\author{
Wang $\operatorname{Min}^{1}$ and He Yan $^{1}$ \\ ${ }^{1}$ Guangxi Medical University First Affiliated Hospital
}

July 23, 2020

\begin{abstract}
Aims The study was to investigate the effects of Intravenous Nicorandil on blood pressure and drug safety in patients with coronary heart disease. Methods In order to evaluate the blood pressure lowering effects and safety of Intravenous Nicorandil, we recruited 43 inpatients with unstable angina to intravenously pump Nicorandil, and the pumping speeds $(2 \mathrm{mg} / \mathrm{h}, 4 \mathrm{mg} / \mathrm{h}$ and $6 \mathrm{mg} / \mathrm{h}$, respectively) were adjusted according to the patient's blood pressure. After treatment, the effects of Intravenous Nicorandil on blood pressure and drug safety were observed. Results After treatment, the average reduction in SBP was $22.86 \pm 19.83 \mathrm{mmHg}$, and the average reduction in DBP was $9.80+-18.58 \mathrm{mmHg}$. And Nicorandil could decrease patients blood pressure in a concentration-dependent manner. During the following-up, no serious adverse events were observed. Conclusion Intravenous Nicorandil could significantly reduce systolic and diastolic blood pressure in patients without affecting heart rate, and it reduced in depending on concentrations, suggesting that Intravenous Nicorandil may be a new intravenous antihypertensive drug in future clinical application.
\end{abstract}

Efficacy and Safety of Intravenous Nicorandil on blood pressure in patients with coronary heart disease

\section{Min Wang ${ }^{1}$ Yan $\mathrm{He}^{1}$}

( ${ }^{1}$ the Department of Geriatric Cardiology, the First Affiliated Hospital of Guangxi Medical University, Guangxi 530000, China ) Corresponging author: Yan He, Email :heyan20200418@126.com

Supported by Guangxi Youth Science Foundation Project, 2018JJB140299, The effect of Neuregulin 4/ErbB4 on intracellular lipids in cardiomyocytes, 2019/1-2022/12

\section{Aims}

The study was to investigate the effects of Intravenous Nicorandil on blood pressure and drug safety in patients with coronary heart disease.

\section{Methods}

In order to evaluate the blood pressure lowering effects and safety of Intravenous Nicorandil, we recruited 43 inpatients with unstable angina to intravenously pump Nicorandil, and the pumping speeds $(2 \mathrm{mg} / \mathrm{h}, 4 \mathrm{mg} / \mathrm{h}$ and $6 \mathrm{mg} / \mathrm{h}$, respectively) were adjusted according to the patient's blood pressure. After treatment, the effects of Intravenous Nicorandil on blood pressure and drug safety were observed.

\section{Results}


After treatment, the average reduction in SBP was $22.86 \pm 19.83 \mathrm{mmHg}$, and the average reduction in DBP was $9.80+-18.58 \mathrm{mmHg}$. And Nicorandil could decrease patients blood pressure in a concentration-dependent manner. During the following-up, no serious adverse events were observed.

\section{Conclusion}

Intravenous Nicorandil could significantly reduce systolic and diastolic blood pressure in patients without affecting heart rate, and it reduced in depending on concentrations, suggesting that Intravenous Nicorandil may be a new intravenous antihypertensive drug in future clinical application.

\section{Keywords}

Intravenous Nicorandil; blood pressure

\section{Introduction}

Angina is one of the common clinical diseases. It is caused by insufficient oxygen supply to the myocardium due to coronary artery stenosis caused by coronary sclerosis or coronary artery spasm. Therefore, reducing myocardial oxygen consumption and increases coronary blood flow and then restores the balance between supply and demand of ischemic myocardial oxygen, at the same time, the prevention and treatment of atherosclerosis also are the fundamentals of angina treatment. Nicorandil is a well-known antianginal agent, which has been recommended as one of the second-line treatments for chronic stable angina as justified by the European guidelines ${ }^{1}$. It shows an efficacy equivalent to that of classic antianginal agents. Nicorandil has also been applied clinically in various cardiovascular diseases such as variant or unstable angina and reperfusion-induced damage following coronary angioplasty or thrombolysis ${ }^{2,3}$. The mechanisms have been involved in the protective effects of Nicorandil in angina through either opening of adenosine triphosphatesensitive potassium (K-ATP) channel or donation of nitric oxide $(\mathrm{NO})^{4,5}$.

Coronary heart disease patients are usually accompanied by hypertension. When experiencing angina pectoris, the patients often had an increase in blood pressure. Clinically, when this happened, intravenous isosorbide mononitrate and nitroglycerin are used to prevent angina, and the same time to control blood pressure. We all know that isosorbide mononitrate and nitroglycerin have anti-angina pectoris and affect blood pressure related to its release of nitric oxide factor ${ }^{5}$. Similarly,Nicorandil administration also increases the level of NO through reaction of its nitrate group with sulfhydryl group in cells of vascular smooth muscle $^{4}$. Therefore, we speculate that Intravenous Nicorandil may also affect blood pressure. The effect of Intravenous Nicorandil on angina is well established,but few reports demonstrated that Nicorandil may cause severe vasodilation and fall in blood pressure, the impact of Intravenous Nicorandil on blood pressure has not been investigated till now.

Therefore, the aim of the study was to investigate the effects of Intravenous Nicorandil on blood pressure and drug safety in patients with coronary heart disease.

\section{Methods}

\subsection{Patients}

This study screened 43 inpatients with unstable angina from the Department of Geriatric Cardiology, the First Affiliated Hospital of Guangxi Medical University from May 1, 2019 to December 1, 2019, The age of included patients was between 39 and 64 years, with a mean age of $51.54+-12.70$ years.

\subsection{Methods}

After admission, patients with unstable angina were given aspirin + clopidogrel or ticagrelor, statins, lowmolecular-weight heparins, and the use of ACEI and receptor blockers to prevent secondary coronary heart disease. Based on this treatment, Nicorandil was intravenously pumped, and the pumping speeds $(2 \mathrm{mg} / \mathrm{h}$, $4 \mathrm{mg} / \mathrm{h}$ and $6 \mathrm{mg} / \mathrm{h}$, respectively) were adjusted according to the patient's blood pressure. We adjusted Nicorandil speed in half an hour, and continuously pumped total of 36mg before stopping the drug. During the treatment, observe the changes in blood pressure and heart rate of the patients before and after 
treatment, and observe whether the effect of Intravenous Nicorandil on the blood pressure of the patients is concentration-dependent, and monitor the adverse reactions during the medication.

\subsection{Statistical analysis}

The SPSS 21.0 statistical software package was used for all the statistical analysis, Continuous variables were expressed as mean +- standard deviation(SD)and categorical variables were presented as frequent count and percentages. The comparison of inter-group analysis of BP levels and HR was performed by unpaired two-tailed t-test. Two-tailed $\mathrm{P}<0.05$ was treated as statistically significant.

\section{Results}

\subsection{Changes in blood pressure and heart rate before and after treatment}

A total of 43 patients were enrolled in this study. After Intravenous Nicorandil treatment, there was induced a significant decrease in levels of SBP and DBP $(\mathrm{p}<0.01)$ (Table 1$)$. After treatment, the average reduction in SBP was $22.86+-19.83 \mathrm{mmHg}$, and the average reduction in DBP was $9.80+-18.58 \mathrm{mmHg}$. In addition, we also compared changes in heart rate before and after treatment ( Table 1), and there was no significant statistical difference $(\mathrm{p}>0.05)$.

\subsection{Nicorandil decreases patient blood pressure in a concentration-dependent manner}

In this study, the intravenous pumping rate of Nicorandil was adjusted according to the patient's blood pressure levels, and the effect of different concentrations of Nicorandil on the patient's SBP and DBP was observed(Table 2). It was found that the systolic blood pressure of the patients can be significantly reduced compared with the baseline blood pressure when the pumping rate of Nicorandil at $4 \mathrm{mg} / \mathrm{h}$ and $6 \mathrm{mg} / \mathrm{h}$, respectively $(\mathrm{p}<0.01)$ ( Figure 1$)$. And the reduction in systolic blood pressure was $18.00+-18.01,27.04+-$ 17.25 respectively. And compared with the baseline DBP, the levels of DBP decreased significantly when the pumping rate of Nicorandil were $4 \mathrm{mg} / \mathrm{h}$ and $6 \mathrm{mg} / \mathrm{h}(\mathrm{p}<0.01)$ (Figure 2). And the reduction in DBP was 8.71+- 9.01 and $15.17+-12.93$, respectively. But when the pumping speed is $2 \mathrm{mg} / \mathrm{h}$, there is no significant statistical changes compared with the baseline SBP and DBP. ( $p>0.05)$ (Figure 1 and Figure 2$)$. These showed that Nicorandil could decrease patients blood pressure in a concentration-dependent manner.

\section{Safety profile}

During the entire observation process, one patient experienced dizziness, two patients experienced headaches, one patient experienced blushing, one patient experienced orthostatic hypotension. All of the above patients were discontinued due to the adverse reactions described above. But, the above symptoms improved after the drug was stopped.

\section{Disscution}

Nicorandil is a safe, well-known antianginal agent that has been approved as a long-term therapy for angina in Japan and Europe ${ }^{6}$. The Japanese Coronary Artery Disease (CAD) and the Impact of Nicorandil in Angina studies have revealed a beneficial impact for Nicorandil on mortality and morbidity in patients with $\mathrm{CAD}^{7,8}$. The use of Nicorandil has been recommended by the European Society of Cardiology as one of the second-line treatments for chronic stable angina ${ }^{1}$. Overall, comparative clinical trials have shown an equivalent efficacy of Nicorandil in improving effort angina and ischemic symptoms compared to $\beta$ blockers and calcium antagonists with minimal hemodynamic disturbance ${ }^{9}$. Interestingly, a meta-analysis from 17 clinical trials has demonstrated that Nicorandil treatment ameliorated left ventricular ejection fraction and microvascular function when used in patients with acute myocardial infarction (AMI) in conjugation with coronary reperfusion therapy ${ }^{10}$.Long-term therapy of Nicorandil also showed beneficial effects on left ventricular remodeling and sympathetic nerve activity of myocardium in patients with AMI when used after reperfusion therapy ${ }^{11}$. In addition, Nicorandil treatment when used as adjunctive to coronary angioplasty was accompanied by better clinical and functional outcomes in patients with anterior AMI compared to angioplasty alone. This was mainly attributed to a decrease in myocardial injury and improvement in microvascular function and rate of no-reflow ${ }^{12}$. Another study revealed that intravenous nicorandil reduced 
QT dispersion and ventricular fibrillation in patients after successful coronary angioplasty ${ }^{13}$. But few studies had repored that Intravenous Nicorandil intake was associated with significant drop in blood pressure. While,in our research, we found Intravenous Nicorandil could significantly reduce systolic and diastolic blood pressure in patients without affecting heart rate, and it reduced in depending on concentrations. Other than that, several reports demonstrated that Nicorandil may cause severe vasodilation and fall in blood pressure when used preoperatively before coronary artery bypass graft ${ }^{14}$. So we can speculate that Nicorandil, like nitroglycerin, may relieve angina and affect blood pressure at the same time.

The vasodilating effect of Nicoradil is relate to the effect of opening the ATP-sensitive $\mathrm{K}$ channel, as well as to the effect of increasing production of cGMP ${ }^{4}$. ATP-sensitive $\mathrm{K}$ channel which not only exists in coronary endothelium, but also in peripheral vascular smooth muscle cells ${ }^{15}$. Probablely,Nicoradil may activate and open ATP-sensitive $\mathrm{K}$ channel of the cell membrane of vascular smooth muscle cells, thus promote the potassium efflux, which leads to vascular smooth muscle cell membrane hyperpolarization. Furthermore, T-type voltage-dependent calciumion channel is closed, reducing intracellular calciumion concentration and inhibiting vascular smooth muscle contraction, therefore affects blood pressure ${ }^{16}$. Meanwhile, Nicoradil can activate guanine cyclase ${ }^{17}$, which could increase cGMP in smooth muscle and other tissues, resulting in dephosphorylation of myosin light chain, regulating vascular smooth muscle contraction, peripheral vein dilatation, blood retention in the peripheral, decreasing re-turned blood volume and left ventricular end diastolic pressure (LVEDP), dilating the artery reduces peripheral resistance. Arteriovenous dilation reduces myocardial oxygen consumption and relieves angina, so it may also decrease blood pressure.

The major adverse reactions of this drug were headache,hepatic function disorder, thrombocytopenia, increased total bilirubin, increased LDH, anemia, and increased $\gamma$-GTPetc ${ }^{18,19}$. In our trial, patients also experienced dizziness, headache, blushing and other discomforts, but the difference is that one patient developed orthostatic hypotension, and its possible mechanism may be related to the rapid infusion of Nicorandil which resulted in a rapid drop in blood pressure.

In our trials, we found that Intravenous Nicorandil had a significant effect in blood pressure lowering and was also safe. Because the sample size of this study is relatively small, and most of our patients are elderly, they may not represent the entire population, so a larger and more comprehensive clinical trial is needed. As the trial progresses, Intravenous Nicorandil may be used, like nitroglycerin, to relieve angina, and the same time, to lower blood pressure. However, we should pay more attention to the pumping speed of nicorandil in the process of using Nicorandil, especially to the elderly, and we must try our best to avoid the risk of orthostatic hypotension.

\section{References:}

1. Montalescot, G., et al. . 2013 ESC guidelines on the management of stable coronary artery disease: the Task Force on the management of stable coronary artery disease of the European Society of Cardiology. EUR HEART J 34, 2949-3003 (2013).

2. Falase, B., Easaw, J. \& Youhana, A. The role of nicorandil in the treatment of myocardial ischaemia. Expert Opin Pharmacother 2, 845-856 (2001).

3. Krumenacker, M. \& Roland, E. Clinical profile of nicorandil: an overview of its hemodynamic properties and therapeutic efficacy. J Cardiovasc Pharmacol 20 Suppl 3, S93-S102 (1992).

4. Refaie, M., Shehata, S., El-Hussieny, M., Abdelraheem, W.M. \& Bayoumi, A. Role of ATP-Sensitive Potassium Channel (KATP) and eNOS in Mediating the Protective Effect of Nicorandil in CyclophosphamideInduced Cardiotoxicity. CARDIOVASC TOXICOL 20, 71-81 (2020).

5. Taira, N. Similarity and dissimilarity in the mode and mechanism of action between nicorandil and classical nitrates: an overview. J Cardiovasc Pharmacol 10 Suppl 8, S1-S9 (1987).

6. Roland, E. Safety profile of an anti-anginal agent with potassium channel opening activity: an overview. EUR HEART J 14 Suppl B , 48-52 (1993). 
7. Walker, A., et al. . Economic evaluation of the impact of nicorandil in angina (IONA) trial. HEART 92 , 619-624 (2006).

8. Horinaka, S., et al. . Effects of nicorandil on cardiovascular events in patients with coronary artery disease in the Japanese Coronary Artery Disease (JCAD) study. CIRC J 74 , 503-509 (2010).

9. Ferrari, R., et al. . Anti-anginal drugs-beliefs and evidence: systematic review covering 50 years of medical treatment. EUR HEART J40 , 190-194 (2019).

10. Shi, L., Chen, L., Qi, G., Tian, W. \& Zhao, S. Effects of Intracoronary Nicorandil on Myocardial Microcirculation and Clinical Outcomes in Patients with Acute Myocardial Infarction: A Meta-Analysis of Randomized Controlled Trials. Am J Cardiovasc Drugs 20 , 191-198 (2020).

11. Kasama, S., et al. . Long-term nicorandil therapy improves cardiac sympathetic nerve activity after reperfusion therapy in patients with first acute myocardial infarction. J NUCL MED 48 , 1676-1682 (2007).

12. Ito, H., et al. . Intravenous nicorandil can preserve microvascular integrity and myocardial viability in patients with reperfused anterior wall myocardial infarction. J AM COLL CARDIOL 33, 654-660 (1999).

13. Ueda, H., et al. . Intravenous nicorandil can reduce the occurrence of ventricular fibrillation and QT dispersion in patients with successful coronary angioplasty in acute myocardial infarction. CAN J CARDIOL 20, 625-629 (2004).

14. Falase, B., Easaw, J. \& Youhana, A. The role of nicorandil in the treatment of myocardial ischaemia. Expert Opin Pharmacother 2, 845-856 (2001).

15. Waldron, G.J. \& Cole, W.C. Activation of vascular smooth muscle K+ channels by endothelium-derived relaxing factors. Clin Exp Pharmacol Physiol26 , 180-184 (1999).

16. Iwaki, F., Amano, H. \& Ohura, K. Nicorandil inhibits osteoclast differentiation in vitro. EUR $J$ PHARMACOL 793 , 14-20 (2016).

17. Li, W., Wu, N., Shu, W., Jia, D. \& Jia, P. Pharmacological preconditioning and postconditioning with nicorandil attenuates ischemia/reperfusion-induced myocardial necrosis and apoptosis in hypercholesterolemic rats. EXP THER MED 10, 2197-2205 (2015).

18. Lee, J.M., et al. . Safety and efficacy of intracoronary nicorandil as hyperaemic agent for invasive physiological assessment: a patient-level pooled analysis. EUROINTERVENTION 12, e208-e215 (2016).

19. Shirakabe, A., et al. . Efficacy and safety of nicorandil therapy in patients with acute heart failure. $J$ CARDIOL 56 , 339-347 (2010).

\section{Hosted file}

Figure 1.docx available at https://authorea.com/users/345459/articles/471678-efficacy-andsafety-of-intravenous-nicorandil-on-blood-pressure-in-patients-with-coronary-heartdisease-corresponging-author-yan-he-email-heyan20200418-126-com

\section{Hosted file}

Figure 2.docx available at https://authorea.com/users/345459/articles/471678-efficacy-andsafety-of-intravenous-nicorandil-on-blood-pressure-in-patients-with-coronary-heartdisease-corresponging-author-yan-he-email-heyan20200418-126-com

\section{Hosted file}

table1.docx available at https://authorea.com/users/345459/articles/471678-efficacy-andsafety-of-intravenous-nicorandil-on-blood-pressure-in-patients-with-coronary-heartdisease-corresponging-author-yan-he-email-heyan20200418-126-com

\section{Hosted file}


table2.docx available at https://authorea.com/users/345459/articles/471678-efficacy-andsafety-of-intravenous-nicorandil-on-blood-pressure-in-patients-with-coronary-heartdisease-corresponging-author-yan-he-email-heyan20200418-126-com 